\section{AKADEMOS}

Órgano de difusión de la Red Docencia-Investigación

\section{ISSN: $1995-4743$}

Año 14 Vol. 1, n. 34 Enero-Junio 2020

AKADEMOS es una revista semestral. De amplio espacio editorial, para la publicación de trabajos inéditos de investigación, artículos de análisis, reseñas y opinión, en los distintos tópicos de las ciencias, la tecnología, las artes y la cultura.

\title{
EI Salvador: Sobre la desigualdad, el COVID-19 y el crecimiento
}

\section{El Salvador: On inequality, COVID-19 and growth}

Mauricio González

Licenciado en Economía e Investigador del Centro de Investigaciones

en Ciencias y Humanidades, CICH de la Universidad Dr. José Matías Delgado

mgonzalezo@ujmd.edu.sv

\section{Resumen}

Aquí se considera i) la relevancia de la desigualdad que surja de la aplicación de una estrategia de crecimiento en El Salvador, $y$ ii) la manera en que se puede integrar el shock del COVID-19 a una estrategia de crecimiento propuesta. Se presenta una conceptualización del crecimiento inclusivo y se distingue entre crecimiento propobre absoluto y relativo, y se presenta la curva de Kuznets relativa al nexo desigualdad/ crecimiento. Se menciona la evidencia internacional y se destaca el caso de China, en el que la desigualdad no es cuestionada en el proceso de crecimiento. En relación con el COVID-19, se mencionan sus probables impactos económicos, se contrastan las respuestas fiscales de los países del centro y la periferia, y se destaca el escaso espacio de política que cabe al país, así como la complicación que reviste la dolarización, en el contexto de la Teoría Monetaria Moderna. Entre los resultados se encuentra que i) la pauta de la desigualdad no es importante en el corto plazo en el crecimiento de El Salvador; ii) los impactos del COVID-19 revertirán significativamente los avances en pobreza y desigualdad; iii) las necesidades de recursos son cuantiosas y empeoran una posición fiscal que ya era delicada; y iv) ante 
la posibilidad de malestar por la manera en que se distribuyen los recursos entre familias, empresas y bancos para la reestructuración económica, se recomienda la elaboración de planes desarrollados por expertos que gocen de consenso.

Palabras clave: desigualdad, crecimiento inclusivo, dolarización, teoría monetaria moderna

\section{Abstract}

Hereweconsider i) therelevance of theinequalitythatarisesfromtheapplication of a growthstrategy in El Salvador, and ii) howthe COVID-19 shock can be integratedinto a proposedgrowthstrategy. A conceptualization of inclusive growthispresented and a distinctionismadebetweenabsolute and relative pro-poorgrowth, and the Kuznets curve relative to inequality / growthnexusispresented. International evidenceismentioned and the case of China, in whichinequalityisnotquestioned in thegrowthprocessishighlighted. In relation to COVID-19, its probable economicimpacts are mentioned, the fiscal responses of thecountries of the center and theperiphery are contrasted, and thescarcepolicyspacethatfitsthe country ishighlighted, as well as thecomplication of dollarization, in thecontext of Modern MonetaryTheory. Amongtheresultsare: i) thepattern of inequalityisnotimportant in the short term in thegrowth of El Salvador; ii) theimpacts of COVID-19 willsignificantly reverse theadvances in poverty and inequality; iii) resourceneeds are large and worsenanalreadydelicate fiscal position; and iv) facedwiththe possibility of discomfortdue to theway in whichresources are distributedamongfamilies, companies and banksforeconomicrestructuring, itisrecommended to draw up plansdevelopedbyexperts with consensus.

Key words: inequality, inclusive growth, dollarization, modern monetary theory.

Pocos temas son de tanta actualidad como el de la desigualdad. Y esto por varias razones. Muchos observadores señalan a la desigualdad como uno de los factores más importantes que explican la ola de malestar político y social que sacude al mundo desarrollado (por ejemplo, Francia), y en desarrollo (Colombia, Perú, Chile).

No obstante, el nuevo protagonista, con un impacto planetario, es el COVID-19. Entre sus efectos, el económico amenaza en convertirse en uno de los peores en la historia de la humanidad.En El Salvador ya se ha tomado una serie de medidas para "aplanar la curva" del virus, incluyendo un probable endeudamiento de $\$ 5.000$ millones, una cifra que aumentaría en 50\% la deuda externa del país.

Con este telón de fondo, vale la pena considerar, primero, la pauta que muestra la desigualdad en El Salvador. Esto se lleva a cabo desde dos ángulos: 1) la importancia que reviste la desigualdad para el crecimiento inclusivo, y 2) las implicaciones de los movimientos entre los quintiles de la distribución del ingreso. 
Luego, en segundo lugar, considerar de modo preliminar la manera en que puede integrarse a una estrategia de crecimiento inclusivo el tratamiento del alivio y superación de los estragos que dejará el COVID-19. Finalmente se concluye.

\section{Desigualdad y crecimiento inclusivo}

El tema de la desigualdad es muy amplio y aquí se procede a una discusión básica en relación con el crecimiento inclusivo, a partir del caso salvadoreño. ${ }^{1} \mathrm{Al}$ respecto, es bien conocida la disyuntiva entre crecimiento y desigualdad. Y en cuanto a la relación entre crecimiento inclusivo y desigualdad, cabe notar que, casi por definición, el crecimiento inclusivo trata, entre otras cosas, de la reducción de la desigualdad a partir de una mayor participación e inclusión de los estratos de menores ingresos de los frutos del crecimiento.

$\mathrm{Al}$ respecto, y como introducción a la consideración del caso salvadoreño, vale la pena referirse a tres situaciones. En primer lugar, cabe mencionar una de las primeras nociones sobre la relación entre desigualdad y el crecimiento inclusivo: la curva $\mathrm{U}$ invertida de Kuznets (1955). En una primera aproximación, la hipótesis es que en las etapas básicas del desarrollo, la desigualdad es creciente; en las etapas intermedias alcanza un máximo, y en las etapas avanzadas se vuelve decreciente; este comportamiento es, precisamente, lo que dota a esta hipótesis con un perfil de $\mathrm{U}$ invertida, porque al principio crece, llega a su máximo, y luego desciende.

Una explicación más detenida señala que al principio ocurre que la mano de obra se concentra en los sectores menos productivos de baja remuneración; luego en el proceso del desarrollo esta se desplaza hacia sectores más productivos de mayor remuneración, lo que se traduce en un alza de la desigualdad por la mayor inversión y acumulación en capital físico que requiere el crecimiento; al final, en una economía madura, la desigualdad cae porque las remuneraciones tienden a una igualación entre los diferentes sectores de actividad económica.

Es probable que China en la actualidad replique este proceso, porque su extraordinario crecimiento ha coincidido con un alza en sus niveles de desigualdad. Pero aquí cabe señalar que el aumento en la desigualdad no parece ser un problema que desvele a nadie en particular, porque coincide con un alejamiento de la pobreza de más de 300 millones de chinos en un período de pocas décadas. De hecho, muchos califican el proceso de crecimiento de China como sencillamente espectacular, como el más llamativo en la era moderna, y uno que se debe emular.

1 Una consideración básica de estos temas, incluyendo su relación con otros como la pobreza y el crecimiento propobre, puede verse, por ejemplo, en lanchovichina and Lundstrom (2009), Ostry et al (2014), y Dabla Norris et al (2015). Una presentación más amplia es la de Piketty (2014). Para el caso salvadoreño puede verse algunos elementos en González (2019) y (2020). 
Solo para recalcar, se trata de un caso en que el aumento de la desigualdad no es preocupante porque ocurre como parte de un proceso de crecimiento económico sumamente veloz y drásticamente reductor de la pobreza en cosa de unas décadas.

En segundo lugar, siguiendo a Ianchovichina y Lundstrom (2009), cabe reflexionar un poco sobre la interacción conceptual entre el crecimiento pro-pobre y la desigualdad a partir de sus definiciones absoluta y relativa:

La definición del crecimiento inclusivo es consistente con la definición absoluta del crecimiento propobre, pero no con la definición relativa. Bajo la definición absoluta, el crecimiento se considera propobre en tanto que la gente pobre se beneficie en términos absolutos, según se refleje en alguna medida acordada de pobreza. ... En contraste, en la definición relativa, el crecimiento es propobre si y solo si los ingresos de la gente pobre aumentan más rápido que los de la población como un todo, i. e., que la desigualdad se reduce. Sin embargo, mientras el crecimiento absoluto propobre puede resultar de esquemas directos de redistribución del ingreso, para que el crecimiento sea inclusivo, la productividad debe mejorarse y crearse nuevas oportunidades de empleo. En breve, el crecimiento inclusivo es acerca de aumentar el tamaño de la economía más bien que redistribuir los recursos. También, el crecimiento inclusivo es acerca de aumentar el ritmo del crecimiento y aumentar el tamaño de la economía, mientras nivela el campo de juego para la inversión e incrementa las oportunidades de empleo productivo.

$\mathrm{Al}$ enfocarse en la desigualdad, la definición relativa podría conducir a resultados sub óptimos, tanto para hogares pobres como no pobres. Por ejemplo, una sociedad que intenta alcanzar un crecimiento propobre bajo la definición relativa querría un resultado caracterizado por un crecimiento del ingreso promedio de 2 porciento donde el ingreso de los hogares pobres creciera en 3 porciento, en vez de un resultado en el que el crecimiento promedio fuera de 6 porciento, pero el ingreso de los hogares pobres fuera de solo 4 porciento. (Págs. 2 y 3 )

En tercer lugar, hay que mencionar que de unas décadas para acá, ha ocurrido una avalancha de literatura en la que se investiga la relación entre desigualdad y crecimiento. En particular, se han examinado las circunstancias desde las cuales las intervenciones redistributivas han conducido a mayor crecimiento económico, y al revés: las circunstancias en que el efecto puede ser el contrario. También ha sido objeto de discusión la identificación del conjunto de políticas que posibiliten el logro conjunto de niveles aceptables de desigualdad y crecimiento.

No deja de ser notable, en este sentido, que en el Fondo Monetario Internacional, FMI, hayan surgido varias contribuciones importantes en esta materia. Por ejemplo, Berg et al (2008), concluyen que 
la duración del crecimiento se halla positivamente relacionada con: el grado de igualdad en la distribución del ingreso; las instituciones democráticas; la orientación hacia las exportaciones (con mayor propensión a la exportación de manufacturas, mayor apertura a la inversión extranjera directa, y la prevención de la sobrevaluación cambiaria que favorece la duración); y la estabilidad macroeconómica (aun con una inestabilidad moderada que pudiera limitar la duración del crecimiento).

Por otro lado, Berg y Ostry (2011), luego que definen un episodio de crecimiento como aquel en que una tendencia de crecimiento lento se interrumpe para dar lugar a un crecimiento mucho más rápido durante un período para luego volver a la tendencia anterior, mencionan lo siguiente:

Encontramos que los episodios de crecimiento largos se asocian de manera robusta con una mayor igualdad en la distribución del ingreso. Por ejemplo, la reducción a la mitad de la brecha de desigualdad entre América Latina y el Asia emergente, provocaría, según nuestras estimaciones centrales, una duración en los episodios de crecimiento de más del doble de lo esperado. La desigualdad típicamente cambia solo lentamente, pero un número de países en nuestra muestra han experimentado mejoras en la distribución del ingreso de esta magnitud en el transcurso de un episodio de crecimiento. Más todavía, la desigualdad sigue siendo importante aun después de tomar en cuenta otros determinantes de la duración de los episodios -como los shocks externos, el ingreso inicial, la cali- dad institucional, la apertura comercial, y la estabilidad macroeconómica.

Los autores pasan a considerar las consecuencias de política económica de su trabajo:

Una implicación clave de nuestros resultados es que es difícil separar el análisis del crecimiento y el de la distribución del ingreso. El papel de la prescripción de política, sin embargo, no es claro. Un aumento de la desigualdad puede limitar la duración del crecimiento, pero los esfuerzos mal diseñados para reducir la desigualdad podrían distorsionar gravemente los incentivos y socavar el crecimiento, lo que también daña a los pobres. Es posible hallar, no obstante, algunas políticas "ganar-ganar", como los subsidios mejor focalizados, mejoras en las oportunidades económicas para los pobres, y políticas activas en el mercado laboral que promuevan el empleo. Cuando hay disyuntivas entre los efectos potenciales de corto plazo de las políticas sobre el crecimiento y la distribución del ingreso, la evidencia presentada en esta nota no es decisiva, pero el análisis adelante parece inclinar la balanza hacia la noción de que la atención a la desigualdad puede traer beneficios significativos de largo plazo para el crecimiento. En los horizontes de largo plazo, la reducción de la desigualdad y el crecimiento sostenido pueden llegar a ser dos lados de la misma moneda. Berg y Ostry (2011)

Los mismos autores, agregando a G. Tsangarides, continúan investigando la relación entre crecimiento económico y desigualdad, y en Ostry et al (2014), a partir de una base 
de datos relativamente abarcadora, llegan a algunas conclusiones que proporcionan luces valiosas para el examen del caso salvadoreño. Ellos concluyen así:

Primero, la desigualdad sigue siendo un determinante poderoso y robusto tanto del ritmo del crecimiento de mediano plazo como de la duración de los episodios de crecimiento, aun controlando por el tamaño de las transferencias redistributivas. Así, las conclusiones de Berg y Ostry (2011) se robustecen y fortalecen. Sería un error enfocarse en el crecimiento y dejar que la desigualdad cuide de sí misma, no solo porque es éticamente indeseable, sino también porque el crecimiento resultante puede ser bajo e insostenible.

Y segundo, sorprendentemente, hay poca evidencia de que la redistribución fiscal implique efectos destructivos en el crecimiento a un nivel macroeconómico. Nosotros encontramos alguna evidencia mixta de que redistribuciones muy grandes pueden tener efectos negativos directos sobre la duración del crecimiento, de manera que el efecto global -incluyendo el efecto positivo sobre el crecimiento por la menor igualdad-puede ser más bien neutral. Pero para redistribuciones no extremas, no hay evidencia de algún efecto directo adverso. Entonces, la redistribución promedio, y la consecuente reducción en la desigualdad, se asocia con un crecimiento mayor y más durable.
Ahora bien, a la luz de estas consideraciones, cabe preguntarse hasta dónde debe llegar la preocupación en El Salvador por el tema de la desigualdad en el contexto de un proceso de crecimiento económico. Algunas preguntas que pueden surgir son las siguientes: Dada la etapa actual de desarrollo ¿Se debe preferir un crecimiento económico propobre según la definición relativa o la absoluta? ¿Qué tanto se debe privilegiar la desigualdad vis-à-vis el crecimiento económico? Finalmente, ¿Qué puede hacerse para mejorar los logros en pobreza y desigualdad e impedir una reversión?

\section{Los datos de El Salvador}

Los datos básicos en desigualdad son los del siguiente cuadro, en el que se distinguen dos períodos: el de 1985 a 2000, de pre dolarización, y el de 2000 a 2017, de pos dolarización. El cuadro muestra los quintiles de la distribución del ingreso, Q1,..., Q5, el coeficiente de Gini, y la participación de la clase media Pcm, que aquí se ha definido como la suma de los quintiles 2 y 3 ; es decir, Pcm $=$ Q2 + Q3. En su mayoría los datos provienen de los World Development Indicators, WDI, del Banco Mundial. A su vez, el Gráfico1 muestra la evolución de la distribución del ingreso para el período 1985 a 2017. 
Cuadro 1

\begin{tabular}{r|rrrrrrr}
\hline \multicolumn{7}{|c}{ El Salvador: cambios en desigualdad, 1985-2000 vrs. 2000-2017 } \\
\hline \multicolumn{7}{|c}{ Puntos porcentuales } \\
\hline Años & Q1 & Q2 & Q3 & Q4 & Q5 & Gini & Pcm \\
\hline $2000-1985$ & 1.15 & 1.90 & 1.36 & 2.46 & -6.88 & -6.50 & 3.26 \\
\hline $2017-2000$ & 3.20 & 3.20 & 3.00 & 1.70 & -11.10 & -13.50 & 6.20 \\
\hline
\end{tabular}

Fuente: elaborado a partir de información de los WDI y retropolaciones.

De inmediato se nota lo siguiente. En relación con el quintil 1, Q1, la clase baja, se observa que su participación aumentó más después de la dolarización que antes de esta: los aumentos son de 3.20 y 1.15 pp, respectivamente. Estos aumentos, con la probable excepción delos del período de pre dolarización, no pueden atribuirse a un mayor crecimiento económico que haya resultado de la dolarización, porque dicho crecimientoha sido muy lento.

A su vez, en González (2020) se argumenta que los cambios en todos los quintiles pueden verse como uno de los principales impactos de las remesas familiares sobre la desigualdad en el país. En el caso del Q1, el canal o mecanismo que se pone de manifiesto es el siguiente. Los trabajadores de este segmento son en general no calificados, y a medida que la población ha experimentado un aumento de la escolaridad, se ha reducido la oferta de trabajo no calificado.

Por otro lado, la demanda de trabajo no calificado ha aumentado como resultado del aumento en la producción de bienes no transables en la economía, que resulta a su vez del mayor gasto en consumo (especialmente bienes no transables) que efectúan los receptores de remesas, quienes ven aumentados sus ingresos por dos conceptos: el primero, un aumento directo, por su condición de receptores de transferencias; el segundo, un aumento indirecto, como asalariados que son contratados por productores de bienes no transables, intensivos en trabajo no calificado.

$\mathrm{Al}$ respecto se recuerda que las remesas son cuantiosas en el país, alrededor de un 16\% del PIB; este es el aumento directo. Y que la masa salarial que se deriva del aumento en la producción no transable, el aumento indirecto, es también considerable, lo señala el Departamento de Economía de la UCA (2019, Págs. 109-115), de cerca de un 12\% de la masa salarial total de la economía, derivado de la generación de casi medio millón de nuevos puestos de trabajo.

Por otro lado, la historia de Q2, Q3 y Q4 es parecida a la de Q1, quizás con menos intensidad a medida que se sube en la es- 
cala de ingresos. Pero con Q5la clase alta, los procesos envueltos, aunque vinculados siempre con las remesas, son diferentes.
Nótese del Gráfico 1 que la tendencia de Q5 es descendente, en agudo contraste con las otras tendencias, que son ascendentes.

\section{Gráfico 1}

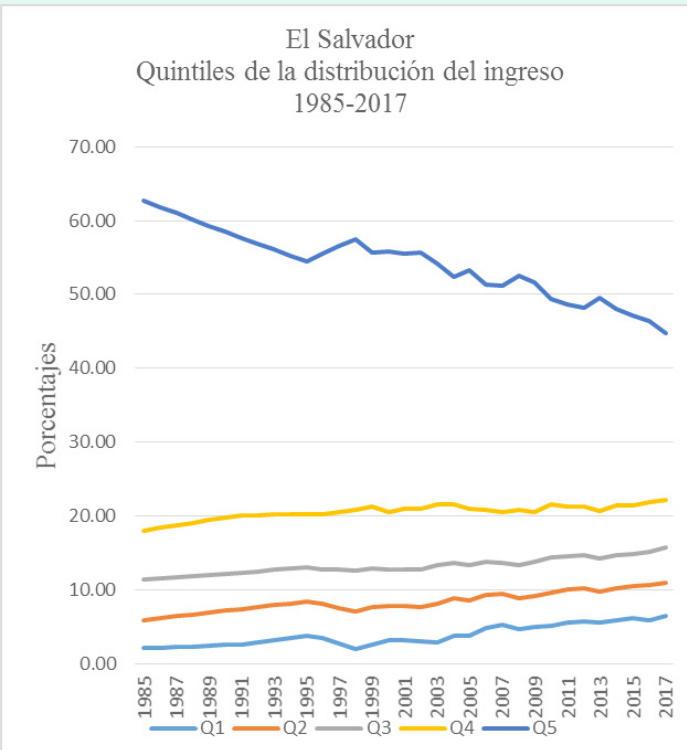

Fuente: elaborado a partir de los WDly otras estimaciones

Lo que ha sucedido en este caso es lo siguiente. ${ }^{2}$ Mientrasla oferta de trabajo calificado se ha reducido y su demanda ha aumentado, con el trabajo calificado, presumiblemente integrado en el quintil 5, sucede exactamente lo contrario: su oferta ha aumentado y su demanda se ha reducido, lo que sugiere una reducción para sus remuneraciones.
La reducción de la demanda de trabajo calificado es como sigue. Al aumentar sostenidamente el caudal de divisas en un país, el tipo de cambio real se aprecia, y los sectores que producen bienes transables pierden competitividad en los mercados internaciones, resultando entonces un estancamiento cuando no una reducción en su producción,

2 Estas explicaciones, con mayor detalle, incluyendo la pauta mostrada por los rendimientos educacionales por nivel de escolaridad, así como la política macroeconómica envuelta, se encuentran en González (2020). 
lo que también conlleva una reducción en la demanda de trabajo calificado.

Una circunstancia adicional es que ante la menor demanda por trabajo calificado, los nuevos trabajadores calificados se ven inducidos a aceptar trabajos no calificados, porque son los que están disponibles -son los que han aumentado-, aunque su remuneración es mucho menor. El impacto distributivo es fuerte. Del gráfico se nota que mientras los otros quintiles ascienden suavemente, el Q5, de la clase alta, desciende de manera pronunciada. De hecho, la importancia que pierde su participación es la que se reparte como ganancia entre los otros quintiles.

Algunos analistas pueden hacer la observación de que tales resultados en el quinto quintil pueden ser engañosos, porque las respuestas de sus integrantes en las encuestas de hogares en todo el mundo adolecen de un sesgo hacia la sub declaración, es decir, a declarar menores ingresos de los obtenidos. $^{3}$

Surgen varias preguntas al respecto. ¿Qué dice la literatura sobre el tema? Desde la perspectiva de un proceso de crecimiento ¿existe alguna pauta sobre la dinámica de la dupla desigualdad/crecimiento en el corto y largo plazos? Y ¿cuáles son las implicaciones para una economía dolarizada? Se ofrecen algunas respuestas a continuación.

3 Es el caso de Departamento de Economía UCA (2019).

\section{Sobre un Gini ajustado}

En ocasiones se considera el caso de un coeficiente de Gini ajustado, con datos que permiten apreciar lo que pasa cuando los datos de las encuestas de ingresos de los hogares o los individuos se complementan con otros datos -los tributarios en particularque permiten efectuar ajustes a los ingresos laborales y agregar los beneficios del capital de la clase pudiente. Una fuente que considera con algún detalle estos aspectos para América Latina esde la Torre et al (2014).

Este trabajo menciona, al analizar el caso de Colombia, que

Algunos estudios recientes han investigado la desigualdad de los ingresos usando registros tributarios administrativos para abordar la representación inadecuada de los más ricos en los datos de las encuestas (Atkinson y Piketty, 2007). Los registros tributarios contienen mucha información sobre los individuos y las empresas, lo que supone un insumo valioso para la mediciónde la desigualdad, pero al mismo tiempo plantea otra fuerte limitación, ya que estos datos no representan adecuadamente al extremo inferior de la distribución. No obstante, los datos tributarios incluyen solo a un porcentaje de la población (bastante pequeño en muchos países en desarrollo) y hay que extrapolar o complementar el resto de la distribución usando datos de otras fuentes.... 
Se extraen dos resultados principales de este ejercicio. El primero, tal vez predecible, es que al incluir información de los más ricos el coeficiente de Gini se incrementa....

En segundo lugar, tener en cuenta a los más ricos no modifica las tendencias a corto plazo de la desigualdad medidas mediante el coeficiente de Gini. Contrariamente a nuestras dudas iniciales (esto es, que el descenso de los coeficientes de Gini de LAC pudiera revertir una vez complementáramos la distribución con los más ricos que nos faltaban), la tendencia sigue siendo buena, aunque tal vez un poco más débil. ...

Su conclusión es como sigue

Dado que la cobertura de los datos de la región es mucho más amplia para las encuestas a los hogares que para los datos tributarios y dado que la variación en los coeficientes de Gini no cambia mucho cuando se implementa la corrección de los ingresos más altos, en lo sucesivo centramos nuestro análisis en medidas de la desigualdad basadas en datos de encuestas. (de la Torre et al,Págs. 39-41)
En realidad, al menos en el caso de la desigualdad en América Latina, en su gran mayoría, la literatura examina el tema a partir de los coeficientes de Gini de losingresos laborales; ocasionalmente se mencionan otros indicadores como los coeficientes de Theil y Atkinson, referidos también a los ingresos laborales. No se encuentra una preocupación importante por la inexactitud en la información en el quinto quintil. En cambio, más bien se acepta que independientemente de si la medición se lleva a cabo con el Gini corriente o el ajustado, el caso es que en América Latina, con la excepción de Costa Rica, ha ocurrido una reducción significativa en la desigualdad en lo que va de los 2000 .

Más allá de lo importante que puede ser la medición de la desigualdad en el país con un Gini ajustado, cabe mencionar que existen evidencias de que en los 2000 la reducción en la desigualdad puede verse como un proceso en el que el quinto quintil se ha visto afectado de forma adversa. Considérese el estudio de Stampini et al (2015). 
Cuadro 2

\begin{tabular}{|l|c|c|c|c|c|c}
\hline \multicolumn{7}{|c}{ Matriz de transición de la pobreza en El Salvador (2003-2013) } \\
\hline & \multicolumn{7}{c}{ Probabilidades de transición } \\
\cline { 2 - 8 } & Pobres & Pobres & Clase & Clase & Ingresos & \\
\hline \multicolumn{1}{|c|}{2003} & extremos & moderados & vulnerable & media & altos & Total \\
\hline Pob. extr. & 61.8 & 30.0 & 8.0 & 0.1 & 0.1 & 100.0 \\
\hline Pob. mod. & 19.9 & 42.3 & 37.1 & 0.6 & 0.0 & 100.0 \\
\hline Cla. vul. & 4.4 & 19.6 & 69.2 & 6.9 & 0.0 & 100.0 \\
\hline Cla. med. & 0.6 & 3.1 & 47.3 & 48.7 & 0.3 & 100.0 \\
\hline Ing. altos & 0.0 & 0.9 & 3.3 & 90.7 & 5.0 & 100.0 \\
\hline
\end{tabular}

Fuente: Stampini et al (2015), pg. 12.

Se trata de una matriz de transición; de transición o movilidad entre clases sociales. Entre otras cosas, dice lo siguiente sobre la clase media en el pais;del 100\% de los que pertenecían a esta clase en 2003: un 0.6\% descendió a la pobreza extrema en 2013; un $3.1 \%$ pasó a la clase de pobres moderados en 2013; un $47.3 \%$ descendió a la clase de vulnerables en 2013; un 48.7\% permaneció en la clase media en 2013; y un 0.3\% llegó a formar parte de la clase alta en 2013.Estos porcentajes pueden interpretarse como las probabilidades de transitar de una clase social en un momento inicial, a otra o la misma clase social en un momento terminal.

También, en el caso de la clase alta, del $100 \%$ de los que pertenecían a esta clase en 2003: un $0.0 \%$ descendió a la pobreza extrema en 2013; un $0.9 \%$ pasó a la clase de pobres moderados en 2013; un 3.3\% des- cendió a la clase de vulnerables en 2013; un 90.7\% descendió a la clase media en 2013; y un 5.0\% permaneció en la clase alta en 2013.

Puede notarse que las probabilidades de transicion son de tres tipos: las de descenso en la escala social -de $51.0 \%$ (¿Por qué?) para la clase media y $95.0 \%$ para la clase alta-; depermanencia-de $48.7 \%$ para la clase media y de $5.0 \%$ para la alta-; y de ascenso en la escala social -de $0.3 \%$ para la clase media y de $0.0 \%$ (¿Por qué?) para la alta.

Una pregunta que surge es ¿Qué tan significativa es la probabilidad de permanencia de la clase alta? Una respuesta se obtiene al observar la misma probabilidad en el caso de los otros paises latinoamericanos según el trabajo de Stampini et al (2015), como se ve del siguiente cuadro. 
Cuadro 3

\begin{tabular}{|l|r|r|}
\hline \multicolumn{2}{|c|}{ Clase alta en países latinoamericanos: 2000-2013 } \\
\hline \multicolumn{2}{|c|}{ Probabilidades de transicion } \\
\hline & Clase media & Permanencia \\
\hline País & 33.3 & 66.7 \\
\hline Argentina & 64.9 & 34.4 \\
\hline Brasil & 25.8 & 74.2 \\
\hline Colombia & 49.2 & 49.7 \\
\hline Costa Rica & 83.2 & 13.0 \\
\hline Rep. Domini & 71.9 & 27.9 \\
\hline Ecuador & 40.6 & 53.1 \\
\hline Honduras & 59.6 & 40.3 \\
\hline Panamá & 69.5 & 30.5 \\
\hline Perú & 47.9 & 50.0 \\
\hline Paraguay & 90.7 & 5.0 \\
\hline El Salvador & 44.7 & 55.3 \\
\hline Uruguay & 58.9 & 40.4 \\
\hline Am. Lat. & \\
\hline
\end{tabular}

Fuente: Stampini et al (2015), Anexo 4.

Se nota que en el período de 2003 a 2013, todas las probabilidades de permanencia de la clase alta en América Latina son menores del $100 \%$, y la menor de todas es la de El Salvador, de 5.0\%.De hecho, en el mismo período el $90.7 \%$ de la clase alta experimentó un descenso en su status social al pasar a formar parte de la clase media en 2013. Esto significa que la clase alta en El Salvador ha sido la más negativamente afectada en el período 2003 a 2013 entre todos los países del cuadro.

\section{Devaluación y desigualdad}

Al examinar la relación entre desigualdad y crecimiento en El Salvador, es conveniente dar atención a un episodio sobre este tema. Es el de la devaluación del peso argentino en 2001. De la Torre et al (2014), al considerar la importancia de la diferencia entre el Gini simple y el ajustado, explican lo que sucedióen los casos de Colombia y Argentina.

Después de concluir que en Colombia existe una diferencia entre estos indicadores, 
pero que la tendencia es la misma para los dos, señalan que:

Estos resultados contrastan con los obtenidos para Argentina durante un periodo que incluye los años de la crisis 2001 y 2002. Aunque los coeficientes de Gini con y sin datos fiscales [Gini ajustado] evolucionan en paralelo en los momentos de calma, hay una divergencia visible durante los tiempos de crisis (el periodo que va de 2001 a 2003), cuando los ingresos de los más ricos aumentaron significativamente más que los del resto. El caso de la crisis argentina es interesante por ser atípico (o, mejor dicho, típico de unas condiciones muy específicas). En 2001, Argentina llevaba diez años con una relación cambiaria fija con el dólar estadounidense $y$, en parte debido a ello, se encontraba financieramente dolarizada, de manera que los hogares de altos ingresos con capacidad para ahorrar tenían una posición larga en dólares (a diferencia del gobierno y las empresas, que tenían una posición corta endólares). Entonces, la devaluación real del 200 por ciento del año 2002 que siguió al colapso de la relación cambiaria fija generó una ganancia de valoración en pesos para estos hogares que compensó con creces el efecto negativo de la crisis sobre los ingresos laborales y sobre el peso relativo de los ingresos laborales en el ingreso nacional. (de la Torre et al, 2014, Págs. 38-39)
Vale la pena explicar esto un poco más. Por partes. En 2002 Argentina tenía un tipo de cambio nominal de 1 peso por 1 dólar. En la crisis, los que pudieron pusieron sus ahorros en dólares, de modo que con la devaluación del 200\% el valor de sus ahorros experimentó un aumento fuerte, de un valor inicial de -por ejemplo- 100 pesos, pasó a 300 pesos. Su tenencia de activos en moneda extranjera se benefició de la devaluación; estaban en posición larga. ${ }^{4}$

En cambio, la deuda en moneda extranjera de las empresas y el gobierno los ponía en posición corta, y resultaron perdedores con la devaluación, porque la deuda aumentó (en moneda doméstica). Además, en la crisis los ingresos laborales se redujeron, y el resultado final fue un Gini (ajustado) mayor que el de predevaluación.

¿Por qué es importante este episodio argentino para El Salvador? Este autor ha recomendado repetidamente el retorno a un régimen cambiario con moneda doméstica, pero de naturaleza flexible, no fijo; a ser seguido de un programa de depreciaciones que permitan al sector exportador recuperar un grado importante de competitividad que se ha perdido por décadas.

Lo que sucedió en Argentina con la devaluación en el caso de los ahorros en dólares de la clase alta sugiere que algo muy parecido

4 Desde luego, los conceptos de "posición corta" y"posición larga", propios de la economía bursátil y financiera, tienen muchas aplicaciones. Aquí es suficiente decir que los ahorros de la clase alta los puso en una posición larga, y que la deuda externa de empresas y gobierno los puso en posición corta, en relación con la devaluación. 
podría suceder en El Salvador: la reducción de riqueza experimentada por la clase alta en los 2000 podría ser compensada por las ganancias de valoración que ante una devaluación podrían experimentar sus ahorros en dólares. Y obvio: a mayor devaluación, mayor el monto de la compensación.

¿Cuáles serían los efectos en el crecimiento y la desigualdad? Comenzando con el primero, del caso argentino se recuerda que con las depreciaciones cambiarias -y los precios al alza de las materias primas- las exportaciones se dispararon yel crecimiento económico fue mucho mayor. Luego, los ingresos laborales aumentaron y el incremento inicial de la desigualdad inducido por la devaluación comenzó a disiparse, aunque el Gini se mantuvo en niveles algo por arriba de los previos a la devaluación.

Si en El Salvador ocurriera algo parecido, en el corto plazo la desigualdad sería mayor, a medida que aumenta la riqueza de la clase alta por la revaloración de sus ahorros por la devaluación; más adelante, comenzaría a reducirse a medida que aumentan los empleos y por ende los ingresos de los trabajadores, también a causa de la devaluación.¿Cómo armonizar esta posibilidad con una estrategia de recuperación y crecimiento inclusivo sostenido?

En otros trabajos ${ }^{5}$ este autor ha sugerido una estrategia de dos componentes básicos:1) una acción macroeconómica, deva- luación-después de desdolarizar, obvio-, a la par de una política industrialen los términos indicados en González (2020); y 2) la puesta en marcha de un programa de empleador de última instancia, EUI, (González, 2018) por el que se persigue el logro del pleno empleo. A estas referencias se remite al lector interesado.

Regresando al tema de la desigualdad, puede surgir la pregunta de qué tan complicada puede ser su agudización en el corto plazo, inmediatamente después del lanzamiento de los componentes mencionados, la devaluación y el EUI. De la discusión en las secciones previas debe ser claro que, en general, no es importante el nivel que tome el coeficiente de Gini, al menos en el corto plazo, mientras la economía esté creciendo y los ingresos laborales también.

Recuérdese el argumento de la curva U de Kuznets: es posible que al principio de un proceso de crecimiento la desigualdad aumente, y que después, a medida que se sostiene el crecimiento, empiece a declinar. Es el caso de China y otros países asiáticos en la actualidad. Y en este caso, la desigualdad no parece ser un tema que inquiete a mucha gente. De cualquier manera, para la etapa de desarrollo del país, las prioridades pueden ser la pobreza primero y la desigualdad después, es decir, resolver el problema de la pobreza antes que el de la desigualdad. 
El comentario de Damill y Frankel (2012) es que:

Si se pidiera a los macroeconomistas que seleccionaran las mejores políticasmacroeconómicas para aumentar el bienestar de un país en desarrollo, una gran proporción de respondentes (incluyendo los autores de este estudio) indicarían que deben ser medidas que induzcan elevadas tasas de crecimiento del producto, empleo y productividad de una manera sostenible.

Naturalmente, el crecimiento rápido de la productividad y empleo no asegura que la desigualdad comenzará a declinar naturalmente. China es el ejemplo más reciente donde un proceso de crecimiento con estas características favorables ha sido acompañado por un empeoramiento de los indicadores de desigualdad. Sin embargo, las políticas macroeconómicas de China no se critican sobre esta base, principalmente por la razón de que el rápido crecimiento de la productividad y el ingreso ocurre a la par de un rápido aumento en el empleo, haciendo que los ingresos menores también crezcan, y resultando en un subsecuente alivio en la incidencia de la pobreza. En general, aunque el rápido crecimiento de la productividad y el empleo puede no asegurar una reducción en la desigualdad, esto crea las condiciones que facilitan una mejor distribución del ingreso, porque los recursos están creciendo y porque una mejora en su distribución parece más viable y menos conflictiva en tal contexto.
En el polo opuesto, un país en desarrollo abrumado por bajos niveles de empleo y productividad podría eventualmente tener una mejora en la desigualdad, pero su trayectoria y duración serían restringidas por un bajo crecimiento de la productividad.En este caso el macroeconomista probablemente criticaría las políticas existentes por su incapacidad para contribuir a la promoción del desarrollo y se enfocaría en un esfuerzo conducente a promover el crecimiento de la productividad y el empleo. (Pág.28)

Y ese es, precisamente, el tono de este trabajo.

\section{El último shock: el COVID-19}

Una manera de abordar la irrupción del COVID-19, C19 en adelante, consiste en darle el tratamiento de un shock severo como pocos a la economía. Desde luego, el C19 ha causado una conmoción mundialde múltiples facetas negativas, a cual peor de todas. Aquí se explora -y de forma limitadatan solo la dimensión económica -que no esnecesariamente la peor-, con la pretensión de que las conclusiones pueden ser valiosas para muchos países en desarrollo; aunque los desarrollados no pueden excluirse.

Aquí se procede como sigue: 1) se presenta una caracterización de los impactos económicos del C19, y 2) se presenta una sugerencia para aliviar y superar sus impactos en el marco de una estrategia de crecimiento inclusivo. 


\section{V.1. C19: impactos y tratamientos}

Aunque el período transcurrido es corto, la literatura en el tema crece rápidamente. En Baldwin y Weder eds. (2020), Hausmann (2020) e ILO (2020) se caracteriza el C19 como un choque de oferta (porque reduce la producción) y como un choque de demanda (porque reduce el consumo y la inversión). De hecho, las acciones de contención del virus como los cierres de comercios y fábricas afectan directamente la oferta, y el despido de empleados y la consecuente reducción de ingresos reduce directamente la demanda. Desde luego, esto en una primera ronda de impactos; los impactos en oferta y demanda continúan en rondas sucesivas. ${ }^{6}$

Una fuente que privilegia el impacto en los mercados laborales y el empleo es el de ILO (2020). Tal perspectiva la comparte este artículo, dada la doble naturaleza del trabajo como agente que genera ingresos y gastos. Entre otras perspectivas también puede verse el trabajo y el empleo como una variable fundamental para el tratamiento de la pobreza y la desigualdad.

En ILO (2020, Pág. 8), se presenta el siguiente esquema, bajo el título

Marco de política: Tres pilares clave para encarar el COVID-19 basado en estándares laborales internacionales
Protección de los trabajadores en el lugar de trabajo

* Fortalecer las medidas de Seguridad y Salud Ocupacionales, SSO

* Adaptación de las formas de trabajo (p. ej., teletrabajo)

* Prevención de la discriminación y la exclusión

* Provisión de facilidades sanitarias para todos

* Expansión de las licencias de trabajo remuneradas

\section{Estímulos a la economía y la demanda} de trabajo

* Política fiscal activa

* Política monetaria acomodaticia

* Préstamos y apoyo financiero a sectores específicos, incluyendo el sector salud

\section{Apoyo al empleo y los ingresos}

* Extender la protección social a todos

* Retención del empleo -trabajo de tiempo parcial, licencias de trabajo remunerado, otros subsidios

* Alivios tributario/financieros para las micro, pequeñas y medianas empresas, MPME

La mayoría de países, unos más otros menos, están aplicando este esquemabásico, incluso El Salvador. Desde luego, este esquema es uno en el que la emergencia es la idea dominante. Así, para el propósito de este estudio, el caso llega a ser la manera en que tal esquema se vuelve parte de la propuesta de crecimiento.

6 Varias estimaciones de impactos en, por ejemplo, PIB, consumo, inversión y empleo, pueden verse en Baldwin y Weder eds. (2020), Hausmann (2020) e ILO (2020). 
Al respecto, cabe mencionar que la propuesta ya cuenta con un componente de emergencia: la puesta en marcha del programa EUI, empleador de última instancia. Esto, entre otras razones, por la falta de empleo en el país. A su vez, la provisiónde empleo ha de contener significativamente los actos delincuenciales y de migración.

Sin embargo, la aparición del C19 sencillamente ha agudizado la urgencia, no solo por su efecto destructor de puestos de trabajo -con un impacto mayúsculo en los empleos no calificados e informales-, sino también en la urgencia de transferir dinero a los desempleados -los nuevos y los de pre C19-. Por esto, la entrega de $\$ 300$ no puede sustituirse por otra acción, al menos en los primeros momentos del C19. Aunque solo sea porque el EUI implica un período de puesta en marcha, no puede sustituir a la entrega de efectivo al comienzo de los enormes y súbitos destrozos causados por el C19.

Solo que aquí cabe mencionar dos aspectos. El primero es el de notar que si bien en el período inmediato al C19 es imprescindible la entrega de efectivo, semejante medida no puede sostenerse más allá de unos cuantos meses, aun contando con apoyo financiero de la comunidad internacional, dado el prácticamente nulo espacio fiscal del país.

El segundo es que, si bien la aplicación de un EUI también requiere ayuda externa, genera empleo de forma directa, mientras que la entrega de efectivo no lo hace. Hay que decir que ambas medidas generan empleo de forma indirecta, al aumentar la demanda agregada cuando se gastan las transferencias en el caso de los $\$ 300$, y cuando se gastan las remuneraciones en el caso del EUI.

¿Por qué es importante la generación directa de empleo en este contexto?Por varias razones. Una es que para la autoestima no es lo mismo vivir de un subsidio que de un salario. Otra es que para la economía es vital, porque la generación de empleo se acompaña de la generación de bienes y servicios directamente generados, porque estos hacen que las sucesivas rondas del proceso productivo, como se conoce del insumo-producto, sean de mayor dimensión; esto porque, a diferencia de la transferencia de efectivo -que solo genera empleo por el efecto indirecto al aumentar la demanda-, genera empleo también de forma directa-además de la forma indirecta-.

\section{V.2. C19 y crecimiento inclusivo}

¿Qué cambios habría que hacer en la estrategia básica de crecimiento que se ha propuesto, para tomar en cuenta la presencia del C19? En realidad, la estrategia sigue igual, pero se agregarían algunas medidas relativas al C19. Esto, en el supuesto de que las infecciones y muertes del virus no pasen hasta el otro año. Con todo, hay que tener en cuenta que el problema que se maneja se ha profundizadocon el C19.

Así, a modo de repaso, se registra una pérdida fuerte de empleos y producción de bienes y servicios. Se ha efectuado un gasto fuerte en transferencias que puede significar algo más de \$400 millones según los medios, y 
se contempla un endeudamiento adicional de $\$ 5000$ millones, es decir, la mitad de la deuda externa del país.

Como se mencionó, el espacio fiscal del país es muy limitado, y la única forma de manejar los gastos de la emergencia es con más endeudamiento externo y/o con la ayuda internacional. Adelante se sugiere una opción, sus pros y cons,que podría reducir sustancialmente las necesidades de financiamiento externo, pero antes conviene considerar algunos aspectos en la manera en que estos problemas se están enfocando en el mundo, desarrollado y en desarrollo.

Lo primero que sobresale en el abanico de acciones de política económica es la utilización del gasto público en magnitudes sencillamente inéditas para la historia económica contemporánea.A la cabeza, en EE. UU.,se ha aprobado un gasto inicial de más de $\$ 2$ millones de millones (aquí será $\$ 2$ billones) para el apoyo del empleo y la producción, y se estima que todavía se solicitarán otros $\$ 5$ billones para los mismos y otros propósitos. En Reino Unido, Alemania, Japón y China, también se mencionan apoyos billonarios al empleo y la producción. Además, a diferencia de lo que sucede en tiempos normales, lo que suceda en términos de mayores déficits fiscales y su impacto inflacionario no se percibe como una preocupación.

En el caso de los países en desarrollo, más allá de que también aumentarán sus déficits fiscales con los mismos objetivos que en los países ricos, se advierte cierta preocupación por la aparición de presiones inflacionarias en la medida en que se expresa preocupación por la obtención de recursos externos después de alcanzar los límites del endeudamiento interno.Por esta y otras razones, varias voces calificadas del primer mundo se han alzado para reclamar suficiente solidaridad de parte de los países industriales para hacer disponibles las cantidades necesarias en condiciones "blandas" para el apoyo a países que el FMI ha denominado "vulnerables". Stiglitz (2020), por ejemplo, sostiene que "la pandemia de COVID-19 es un problema global que exige una solución global".

En realidad, en este momento, esta es la única opción que tiene el país. Es decir, sus posibilidades de más endeudamiento en los términos usuales, con un riesgo país que es ahora mayor como consecuencia del C19, en montos de varios miles de millones de dólares, sencillamente no existe, o existe pero de forma muy limitada en función de las magnitudes que se necesitan. El país debe recurrir a la solidaridad internacional que se forme alrededor de este problema -podría pedirse al FMI y al Banco Mundial que la administren-, porque la mayor parte del mundo en desarrollo se halla igual o peor.A diferencia de los países centrales, el tercer mundo, en gran parte,no podrá resolver la emergencia sin la solidaridad de aquellos. 


\section{V.2.1. Sobre la TMM en países centrales y periféricos}

No solo es el caso de que las acciones necesarias para recuperar cierta normalidad se hallan fuera del alcance de lo que el país puede hacer porque carece de espacio de política económica, especialmente espacio fiscal; se trata también de que los países del centro se han visto desbordados en la utilización de sus instrumentos convencionales de política económica -la manipulación de la tasa de interés de política por los bancos centrales, como sucede en la UE-, para encarar las consecuencias económicas de la peste, y se han visto obligados a recurrir a medidas no convencionales, en un proceso que traslada el protagonismo de la política monetaria, según la macroeconomía tradicional, a la política fiscal, mediante el recurso del gasto masivo, sin mayores preocupaciones por los aumentos de deuda pública, la inflación o el déficit fiscal. La lógica dominante es la de "hacer lo que haya que hacer" para superar la paralización económica, en alusión a la célebre frase del expresidente del Banco Central Europeo, (BCE), Mario Draghi, cuando recurrió al gasto masivo para apuntalar la recuperación europea -y de paso salvar al euro de su desaparición- en ocasión de la Gran Recesión de 2008/09.

$\mathrm{Al}$ respecto, conviene señalar lo siguiente. En primer lugar, en los países industrializados, la inflación no se considera una li- mitante importante para dejar de imprimir dinero por billones y hacerlo disponible a través del sistema bancario o, en un esquema más amplio,"arrojarlo desde un helicóptero a las empresas y a la población necesitada", algo parecido a la entrega de $\$ 300$ a las familias en el país.Se recuerda que esto ha sucedido antes al menos en doscasos: en los 1930 con la Gran Depresión, cuando en realidad la inflación hasta se redujo a niveles récord para EE. UU.; y en la Gran Recesión de 2008/2009, cuando se practicaron los "relajamientos cuantitativos" por parte de la FED, dando lugar a la depreciación del dólar y luego a una "guerra de divisas", especialmente contra países emergentes como China y Brasil.

En segundo lugar, resultado de lo anterior, tales episodios de gasto masivo no inflacionario han sido tomados como prueba de que el gasto gubernamental no tiene que ser inflacionario siempre que existan recursos ociosos, especialmente la mano de obra, como sostienen los partidarios de la denominada Teoría Monetaria Moderna, TMM. $^{7}$ En este punto, cabe mencionar algunas implicaciones para el caso salvadoreño.

Efectivamente, se pueden mencionar dos aspectos. El primero hace referencia al grado al que en El Salvador se puede repetir la acción de un gasto masivo no inflacionario enmoneda nacional -no en dólares porque no puede emitirlos-, para minimizar el

7 Una introducción a la TMM, las escuelas de pensamiento que le originan, así como sus vínculos con un EUl y sus implicaciones para la política cambiaria, se halla en González (2018). 
impacto negativo del C19 en la economía salvadoreña al mismo tiempo que se opera la recuperación. Obvio, la dificultad aquí es la dolarización oficial actual del país, de manera que el paso previo sería la desdolarización al mismo tiempo que se establece una moneda nacional, lo cual permitiría una emisión como la de los países grandes, y en principio al menos, no inflacionaria.

No obstante, desde el punto de vista de los adversarios de la TMM, esto puede no ser tan inmediato; incluso podría ser muy difícil. ${ }^{8}$ Edwards (2019), por ejemplo, denominasu puesta en práctica "una receta para el desastre", y sostiene que los episodios de hiperinflación en América Latina, los de Argentina, Chile, Perú y Venezuela entre 1970 y 2019, resultaron por un intento de aplicar la TMM.

Todavía más, entre los detractores de la TMM se mencionan nombres como los de Kenneth Rogoff, Paul Krugman, Carmen Reinhart, etc., y el número está creciendo, probablemente resultado de que los adeptos a la TMM también están aumentando, como se nota del hecho de que algunos de los nombres que se mencionan en la contienda electoral de 2020 en EE. UU., como los de Paul Sanders y Alexandria OcasioCortez, se decantan abiertamente por la TMM para respaldar algunas de sus propuestas como la de una garantía federal de empleo, una propuesta similar a la del EUI.
Una cosa es cierta: el marco analítico que mejor parece explicar la razón por la que el gasto masivo no deriva en inflación, y el aumento en el déficit fiscal y la deuda dejan de ser un problema, es el de la TMM en su concepto de la soberanía monetaria-un país que emite su propia moneda con un reconocimiento internacional-. Más detalles sobre esta noción se encuentran en las referencias al final. Después de todo, los partidarios de la TMM siempre han mencionado los episodios más o menos catastróficos como la Gran Depresión de los 1930, las guerras mundiales, enormes terremotos e inundaciones, y los relajamientos cuantitativos practicados por la FED y el BCE por la Gran Recesión, como ocasiones en que se suele adoptar medidas extraordinarias, apartadas de la lógica económica prevaleciente y a veces hasta en contra de ésta, pero que al final resultan muy consistentes con la TMM. Es por esto que indican que el colapso causado por el C19 es uno cuyo tratamiento efectivo está más allá de lo que se puede hacer con la macro convencional con su énfasis en bajas tasas de inflación y déficit fiscal, mientras aconsejan su manejo en la perspectiva de la TMM, con su énfasis en el empleo, sin descuidar la inflación, $i$. e., sin hiperinflación, como a menudo le han imputado, equivocadamente, sus críticos.

Todavía más. ¿Cómo surge el énfasis en el empleo en el contexto de la TMM? Una manera de verlo es a partir de la aplicación 
de la TMM a situaciones calamitosas: si esta resulta tan apropiada para la gerencia de la Gran Depresión con su New Deal, la Gran Recesión con su Cuantitative Easing, y las guerras mundiales, ¿por qué no aplicarla a situaciones que pueden ser más o menos igual de dramáticas, aunque en tiempos "normales", como el desempleo masivo que campea en gran parte del tercer mundo e incluso en algunos países de la UE?

Es por este y otros argumentos que la TMM llega a ser el entorno natural para el manejo de conceptos tan vinculados al empleo y la pobreza, y aun la desigualdad, como los del EUI y el Ingreso Básico Universal; este último incluso con algún historial en Europa y siendo objeto de propuestas para atender la pérdida de empleo causada por el C19 en varios países.

En la medida en que tantas acciones no convencionales y de un carácter más bien extraordinario se llevan a cabo para un evento también extraordinario, pudiera suceder que más adelante se reconozca la utilidad de la TMM. En Japón, por ejemplo, la deuda pública es algo más de 230\% del PIB, y prácticamente no hay inflación; ante la imposibilidad de aumentar el crecimiento por medio de manipular la tasa de interés se ha incrementado el gasto público. Las autoridades, sin embargo, no reconocen que esto sea seguir los preceptos de la TMM. Lo mismo ha declarado la FED con el Quantitative Easing, que no sigue la TMM. Parece que es algo así como "haz lo que hago, no lo que digo".
Sin embargo, este pudiera ser el momento de la TMM. Como señalan Blanchard y Summers (2017), en relación con la Gran Recesión (o Gran crisis):

¿Por qué elegimos poner "Volver al futuro» en el título de nuestro artículo? Porquevemos que las lecciones básicas de la Gran crisis financiera son similares a las extraídas de la revolución keynesiana en respuesta a la Gran Depresión: las economías puedenverse afectadas por fuertes golpes y no se puede esperar que se estabilicen automáticamente.No tenemos duda de que, en ausencia de las fuertes respuestas de política monetaria y fiscal que se han dado, la crisis financiera habría llevado a un resultado tan malo o peorque el de la Gran Depresión. Por lo tanto, las políticas fuertes de estabilización son simplemente esenciales.

Entonces, en tanto la crisis del C19 se trate con las mismas medidas de política monetaria $y$, especialmente fiscal, que se emplearon en los 1930 y después de 2008, la pregunta será ¿es ahora el momento para reconocer los méritos de la TMM? Probablemente no todavía. Hay muchas críticas desde la ortodoxia y aun desde algunos economistas heterodoxos; además, en particular, es posible que se necesite más investigación en cuanto al grado y ajustes para su aplicación en entornos del tercer mundo, como el salvadoreño.

El manejo cambiario y la restricción externa que la balanza de pagos impone a los esfuerzos para el crecimiento se hallan entre 
los temas principales por los que la TMMno puede aplicarse sin más a las economías en desarrollo. Como si esto fuera poco, el caso salvadoreño adolece de una complicación adicional que muy pocos países padecen: no tiene una moneda propia y por esto no puede emitir las cantidades que se requieren para aliviar el impacto del C19. Tendría que endeudarse de forma extraordinaria y soportar una carga elevada que será una rémora para el crecimiento.

\section{V.2.2. Sobre la solidaridad internacional}

Se sabe que las implicaciones del C19 son peores que las de la Gran Depresión, y que el mundo en desarrollo se verá afectado con mayor dureza, resultado de su insuficiencia de recursos en montos superiores a casi cualquier cifra antes requerida. Es claro que los países pobres no podrían hacer frente a estas necesidades, y los llamados por solidaridad por parte de los países del centro no se han hecho esperar. Vale la pena mencionar la propuesta de UNCTAD (2020): ${ }^{9}$

Ante el inminente tsunami financiero de este año, la UNCTAD propone una estrategia de cuatro frentes que podría comenzar a traducir las expresiones de solidaridad internacional en acciones concretas:

1. Primero, una inyección de liquidez de $\$ 1$ billón; una especie de caída de dinero en helicóptero para quienes se quedan atrás reasignando los derechos especiales de giro existentes en el Fondo Monetario Internacional y agregando una nueva asignación que deberá ir considerablemente más allá de la asignación de 2009 realizada en respuesta a la crisis financiera mundial.

2. En segundo lugar, un jubileo de la deuda para las economías en dificultades. Una suspensión inmediata de los pagos de la deuda soberana debe ir seguida de un alivio significativo de la deuda. Un punto de referencia podría ser el alivio de la deuda alemana administrado después de la Segunda Guerra Mundial, que canceló la mitad de su deuda pendiente. En esa medida, alrededor de $\$ 1$ billón debería ser cancelado este año supervisado por un organismo creado de forma independiente.

3. En tercer lugar, un Plan Marshall para la recuperación de la salud financiado por parte de la asistencia oficial para el desarrollo (AOD) que ha faltadoy que ha sido prometida desde hace tiempo pero no entregada por los socios del desarrollo. La UNCTAD estima que se deben destinar $\$ 500$ mil millones adicionalesuna cuarta parte de la AOD faltante de la última década- en gran parte en forma de subvenciones para ser- 
vicios de salud de emergencia y programas relacionados de ayuda social.

4. Finalmente, los controles de capital deben tener su lugar legítimo en cualquier régimen de políticas para reducir el aumento de las salidas de capital, reducir la falta de liquidez provocada por las ventas en los mercados de los países en desarrollo y detener las caídas en los precios de las monedas y los activos.

El paquete propuesto es similar en tamaño a la cantidad que se habría entregado a los países en desarrollo durante la última década si los países del Comité de Asistencia para el Desarrollo de la Organización para la Cooperación y el Desarrollo Económicos hubieran alcanzado su objetivo de AOD del 0,7\%.

Se trata de una propuesta del tipo de "hacer lo que sea necesario", como en la UE y EE. UU., para atender de manera proporcionada los problemas generados por el C19 en los países pobres. Esto indica que hay conciencia de las necesidades de financiamiento de los países pobres para encarar las consecuencias del C19. Ahora supóngase que esta sensación se traduce en hechos y la deseada solidaridad se materializa, incluso en los montos necesarios que algunos estiman en $\$ 2.5$ billones para el mundo en desarrollo.

Los problemas no terminan ahí. Primero, aun en los países del centro se padece una gran incertidumbre en cuanto a la manera precisa en que continuará desarrollándose el C19: si terminará este año, si habrá rebrotes, de qué magnitud, etc., así como la forma específica en que afectará a los sectores económicos.

Segundo, por la incertidumbre, la preparación de planes para la reactivación -reparto de los recursos que mitigarán los efectos en las familias, las empresas y los bancos por el grave aumento de la morosidad-se lleva a cabo sobre una base incierta; y tercero, el tiempo está en contra de los mejores resultados, porque a medida que transcurre se profundiza la crisis y por esto el costo del confinamiento.

En este punto cabe mencionar las necesidades de legitimización del proceso de asignación y reparto de los recursos -que se sugiere sea construido y administrado por un equipo de expertos desde el Banco Mundial o UNCTAD, buscando imparcialidad- para prevenir cualquier señalamiento que alegue fraudes o favoritismos; especialmente si, como también se sugiere, las asignaciones revisten el carácter de transferencias o subsidios, como se ha planteado para la UE por parte del BCE -O sea, recursos no reembolsablesen vez de préstamos. Entre otras razones, porque si se conceden en calidad de préstamos, habrá un cargo que tendrá que atenderse, complicando la misma recuperación.

Hay que entender que la crisis es de tal magnitud que la recuperación puede significar un nuevo proceso económico y social,y por esto pudiera requerirse una especie de nuevo contrato social que goce de la aceptación de todos los sectores. Entre los criterios de 
priorización para el reparto -porque los recursos con seguridad serán escasos- puede utilizarse el triage: los más graves primero, y según el orden de gravedad se atiende el resto; hasta donde alcance. Esto también requiere que todos entiendan que el Gobierno cargará con todos los costos o su mayoría. (Véase Banco Mundial, 2020). ${ }^{10}$

Todos estos son problemas graves para el centro, y en la periferia pueden ser el doble o el triple o más, con agravantes como los siguientes: 1) las redes de protección social ofrecen una cobertura escasa a una masa laboral en la que la informalidad fácilmente sobrepasa el $50 \%$ de las personas ocupadas; 2) escasa bancarización, lo que dificulta la entrega eficaz de transferencias de efectivo a la población, una de las medidas básicas y esenciales para lidiar con los efectos del C19; y 3) una fuga de capitales desde la periferia al centro del orden de $\$ 100.000$ millones, complicando la necesaria recapitalización para la recuperación del sector empresarial. En el caso salvadoreño agréguese que se espera una importante contracción de las remesas y que por la dolarización no se puede emitir deuda en moneda nacional, y menos intentar probar una estrategia en el marco de la TMM.

En este punto cabe mencionar la alusión que a los controles de capital hace la UNCTAD en la cita anterior. Desde la perspectiva de largo plazo que atañe al crecimiento económico, la vigencia de un tipo de cambio real competitivo (TCRC) es crucial. En varios de nuestros escritos hemos explicado las dificultades que para un país pequeño y abierto reviste la ausencia de los controles de capital para la gestión de tal instrumento como una política de crecimiento, así como para impedir una salida desordenada de capitales como ocurre desde la periferia hacia el centro, lo que puede dar lugar a depreciaciones cambiarias disruptivas. Si a esto se añade una crisis financiera por el aumento de la morosidad, la calamidad está garantizada, al mismo tiempo que se profundizan los problemas que han de dificultar todavía más el crecimiento posterior.

Más todavía. De nuestros escritos se advierte que la vigencia de un TCRC es sencillamente indispensable para el crecimiento inclusivo. Es fácil notar que con las cantidades astronómicas de gasto desde los países del centro vendrá también una marcada depreciación de sus monedas, lo que en principio plantea dificultades mayores para que países como El Salvador dispongan de tipos de cambio competitivos. Esto ya ocurrió en ocasión del Quantitative Easing de la FED, pero también con el gasto masivo de la UE y Japón en ocasión de la Gran Recesión de 2008/2009, desatando lo que algunos llamaron "la guerra de divisas".

10 ¿Puede ver el lector alguna analogía con la TMM? Recuérdese que lo que los Gobiernos están haciendo - gasto fiscal "hasta donde sea necesario", en EE. UU. y la UE- para tratar con el C19, se explica cómodamente con la TMM; aunque, desde luego, estos Gobiernos niegan que la lógica de sus acciones siga la de la TMM. 
Los controles a los movimientos de capital han llegado a ser un instrumento formidable-acompañado por otros como un programa de devaluaciones-para lidiar con aquella eventualidad, como parte del kit de instrumentos macroprudenciales que se han implementado con eficacia comprobada por muchos países de América Latina en los 2000. ${ }^{11} \mathrm{El}$ panorama que se percibe después del C19 señala la elevada probabilidad de que los controles de capital serán reutilizados.

\section{Conclusiones}

En este trabajo intentamos elaborar sobre la incidencia de la desigualdad y el C19 en las posibilidades de crecimiento de El Salvador.

Entre nuestros resultados podemos listar los siguientes. Primero, la relación entre desigualdad y crecimiento no tiene particular relevancia en este país en la etapa actual de desarrollo; podría tenerla después pero no ahora, cuando la desaparición de la pobreza es más urgente.

Segundo, en términos de distribución del ingreso, se constata la nada trivial reducción de la participación de la clase alta -unacaída de 18 pp en el período de 1985 a 2017; y de 11 pp en el período 2000 a 2017, de pos dolarización-con consecuencias negativas para el crecimiento, en la medida en que se reduce significativamente el capital empresarial que más se necesita en las primeras etapas del desarrollo, cuando más que reducir la desigualdad interesa la reducción de la pobreza. La quiebra de empresas que resulte del C19 habrá de empeorar la situación de la clase pudiente.

Tercero, en relación con el C19, sus efectos en las pretensiones de crecimiento económico del país son graves. Se trata de un choque de oferta y uno de demanda, por los que se han roto los encadenamientos entre trabajadores y empresarios que tanto tiempo lleva construir y llevará reparar. Las medidas de confinamiento parecen haber sido eficaces en la contención de la pandemia, pero a un costo elevado explicado por la paralización económica de varios meses. En términos de pobreza y desigualdad se espera una desmejora importante de los logros alcanzados en las últimas décadas.

Cuarto, el país carece del espacio fiscal necesario para encarar la recuperación económica en el contexto de "hacer lo que sea necesario" para minimizar los impactos en el empleo, la producción, y en el sistema financiero, ya que con toda probabilidad se verá afectado ante los impagos de sus clientes.

Quinto, el endeudamiento adicional en que se incurrirá ha de agravar la severidad de la restricción externa al crecimiento económico, a lo que se agrega una segura reducción importante en el flujo anual de remesas familiares.

11 Véase, por ejemplo, de la Torre et al (2013). 
Finalmente, más allá del retorno a los lugares de trabajo y a la actividad económica y su importancia para volver a la normalidad, la obligada reestructuración en los ámbitos de los hogares, las empresas y el sistema financiero, vuelve imprescindible, y por esto recomendable, la elaboración de una estrategia para la recuperación, consistente en un conjunto de planes para llevarla a cabo, de forma efectiva y, principalmente, transparente, como resultado de un trabajo esencialmente técnico llevado a cabo por expertos en la gestión de crisis análogas. Esto debe hacerse desde la perspectiva de un pacto social -a partir de un consensopara la reestructuración económica, que dote de legitimidad al proceso, previniendo así cualesquier dudas y/o denuncias contra el proceso en sí. 


\section{Referencias}

1 Atkinson, A. y Piketty, T. (2007). Top Incomes over the Twentieth Century: A Contrast Between Continental European and English-Speaking Countries, Oxford: Oxford University Press.

2 Berg, Andrew and Jonathan D. Ostry (2011), "Inequality and Unsustainable Growth:Two Sides of the Same Coin?" IMF Staff Discussion Note SDN/11/08. International Monetary Fund. Washington.

3 Baldwin, Richard and Beatrice Weder di Mauro, eds. (2020), Economics in the Time of COVID-19. Centre for Economic Policy Research (CEPR).

4 Banco Mundial (2020), "La economía en los tiempos del COVID-19". Informe semestral de la región de América Latina y el Caribe. Washington D. C. Banco Mundial.

5 erg, Andrew, Jonathan D. Ostry, and Jeromin Zettelmeyer (2008), "What Makes Growth Sustained?" IMF Working Paper WP/08/59. International Monetary Fund. Washington.

6 Blanchard, Olivier and Lawrence Summers (2017), "Rethinking Stabilization Policy. Back to the Future". Peterson Institute for International Economy, PIIE. October 8.

7 Dabla-Norris, Era, Kalpana Kochhar, NujinSuphaphiphat, Frantisek Ricka and Evridinka Tsounta(2015), "Causes and Consequences of Income Inequality: A Global Perspective." IMF Staff Discussion Note SDN/15/13. International Monetary Fund. Washington.

8 Damill, Mario and Roberto Frankel (2012), "Macroeconomic Policies, Growth, Employment, and Inequality in Latin America". UNU-WIDER Working Paper No. 2012/23. UNU-WIDER.

9 de la Torre, Augusto, Eduardo Levy Yeyati, Guillermo Beylis, Tatiana Didier, Carlos Rodríguez-Castelán, y Sergio Schmukler (2014), "Desigualdad en una América Latina con Menor Crecimiento". Informe Semestral, Oficina del Economista Jefe Regional. Banco Mundial. Washington.
10 de la Torre, Augusto, Eduardo Levy Yeyati, Samuel Pienknagura (2013), "La desaceleración en América Latina y el tipo de cambio como amortiguador." LAC Informe Semestral (Octubre), Banco Mundial, Washington, DC

11 Departamento de Economía UCA (2019), "Análisis socioeconómico de El Salvador: un enfoque estructural 1985 - primer trimestre 2019. Universidad Centroamericana José Simeón Cañas.

12 Edwards, Sebastian (2019),"Modern Monetary Theory:Cautionary Tales from Latin America". Cato Journal, Vol. 39, No. 3.

13 González O., Mauricio (2020), El Salvador: Macroeconomía de la Pobreza, la Desigualdad, y el Crecimiento. Centro de Investigaciones en Ciencias y Humanidades, $\mathrm{CICH}$. Universidad Dr. José Matías Delgado.

14 González O., Mauricio (2019), El Salvador: Desigualdad, Clase Media y Crecimiento. Instituto de Ciencia, Tecnología e Innovación, ICTI, Universidad Francisco Gavidia. (No publicado)

15 González O., Mauricio (2018), El Salvador: Empleo y Crecimiento. Instituto de Ciencia, Tecnología e Innovación, ICTI, Universidad Francisco Gavidia.

16 Ianchovichina, Elena and Susanna Lundstrom (2009), "Inclusive Growth Analytics. Framework and Application." Policy Research Working Paper 4851. The World Bank. Washington.

17 International Labour Organization (2020), "COVID-19 and theworld of work: Impactand policy responses". ILO.

18 Kuznets, Simon (1955), "Economic Growth and Income Inequality." American Economic Review 45(1); pp $1-28$.

19 Ostry, Jonathan D., Andrew Berg, Charalambos G. Tsangarides (2014), "Redistribution, Inequality, and Growth." IMF Staff Discussion NoteSDN/14/02. International Monetary Fund. Washington. 
20 Piketty, Thomas (2014), Capital in the Twenty-first Century. Cambridge, Massachusetts. Harvard University Press.

21 Stampini, Marco, Marcos Robles, Mayra Sáenz, Pablo Ibarrarán, y Nadin Medellín (2015), “Pobreza, vulnerabilidad y la clase media en América Latina". Documento de trabajo del BID No. 591.

22 Sumner, S., and Horan, P. (2019) "How Reliable Is Modern Monetary Theory as a Guide to Policy?" Arlington, Va.: Mercatus Center, George Mason University (March 11).

23 Stiglitz, Joseph (2020), "Internationalizing the Crisis". Project Syndicate.

24 Tymoigne, Éric and Wray, L. Randall (2013), "Modern Money Theory 101: A Reply to Critics." Levy Economics Institute, Working Paper No. 778.
25 UNCTAD (2020), "UN calls for \$2.5 trillion coronavirus crisis package for developing countries". UNCTAD, March 30.

26 UNCTAD (2020a), "The COVID-19 Shock toDeveloping Countries:Towards a "whatever it takes"programme for the two-thirdsof the world's populationbeing left behind". UNCTAD/GDS/INF/2020/2, March.

27 Wray, L. Randall (2018), "How I Came to MMT and What Do I Include in MMT." Remarks at the 2018 MMT Conference, September 28-30, New York City.

28 Wray, L. Randall (2015), Modern Money Theory: A Primer on Macroeconomics for Sovereign Monetary Systems. 2nd ed. London: Palgrave Macmillan. 